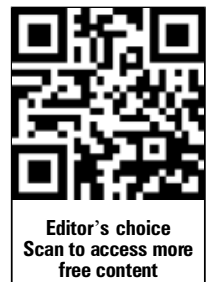

Department of Genito-Urinary Medicine, NHS Trust, University Hospitals Leicester, Leicester, UK

\section{Correspondence to} Dr Adrian Palfreeman, Department of Genito-Urinary Medicine, NHS Trust, University Hospitals Leicester, Jarvis Building, Infirmary Close, Leicester, LE1 5WW, UK; adrian.palfreeman@uhl-tr.nhs. uk

Accepted 29 September 2012 Published Online First 7 November 2012
To cite: Palfreeman $\mathrm{A}$ Nyatsanza F, Farn $\mathrm{H}$, et al. Sex Transm Infect 2013;89:308-310.

\title{
HIV testing for acute medical admissions: evaluation of a pilot study in Leicester, England
}

\author{
Adrian Palfreeman, Farai Nyatsanza, Helen Farn, Graham McKinnon, Paul Schober, \\ Paul McNally
}

\section{ABSTRACT}

Objectives The 2008 UK National Guidelines for HIV testing recommended HIV testing should be offered to all general medical admissions aged 16-60 years in high prevalence areas, and that this should be evaluated to ensure this was effective in diagnosing previously undiagnosed HIV.

Methods HIV testing was introduced as a routine test for all patients admitted to the acute medical admissions unit, comparisons were made between the testing rates before, during and after this intervention.

Results The pilot was initiated in August 2009. Prior to the pilot the unit was carrying out 15 tests per month. However, when the pilot was introduced 82 tests were being carried out per month with a total of 10 new diagnoses since the start of the pilot. The proportion of patients tested versus those eligible for testing remained low varying between $6 \%$ and $22 \%$ month by month. 10 patients we found to be HIV positive with a prevalence of approximately 1\%, 10 fold higher than the cut off for cost effectiveness used in the guidelines.

Conclusions Overall the pilot showed that HIV testing could be delivered without the use of extra resources and is acceptable to patients.

\section{INTRODUCTION}

3In September 2007, the Chief Medical and Chief Nursing Officers sent out a letter to all doctors in the UK calling for 'a special effort' to improve rates of HIV diagnosis. ${ }^{1}$ This appeared to have little impact. ${ }^{2}$

A national audit looking at causes of death in patients living with HIV showed that presenting for care too late for effective treatment was significantly associated with subsequent death. ${ }^{3}$

These HIV infected patients who are unaware of their status are at particular risk of harm as they can present late to care without the diagnosis being immediately apparent to those caring for them, and may have had repeated contact with health care professionals without being offered an HIV test. ${ }^{4}$

In 2008 new National Guidelines for HIV testing ${ }^{5}$ were issued which stated that-

- HIV is now a treatable medical condition with a good prognosis if detected early

- Improving levels of detection will help reduce onward transmission

- HIV testing should be offered as routine in certain clinical settings or when patients present with particular indicator conditions

- It should be within the competence of any doctor, midwife, nurse or trained healthcare worker to obtain consent for and conduct an HIV test.

The guidelines suggested that testing should be considered in all general medical admissions where prevalence is greater than 2 per $1000 .^{6}$

Leicester City had a prevalence of $2.83 / 1000$ so we decided to introduce HIV testing on the acute medical admissions unit at Leicester Royal Infirmary as part of the DH HIV testing pilot scheme.

Permission was sought from the local ethics committee, and we were advised that as this pilot was implementing national guidance no ethics submission was required.

\section{OBJECTIVE}

The objective of this pilot study was to establish whether a routine offer of an HIV test in a busy Acute Medical Admissions unit could be practically implemented without additional resources.

\section{METHODS}

Following discussions with relevant members of the AMU staff including medical, nursing and clinical aides and with the virology dept. the following model was agreed.

We reviewed the number of HIV tests performed on AMU for 12 months prior to the pilot.

We then introduced routine testing of all patients admitted and used the funding from the $\mathrm{DH}$ for the pilot to pay for the laboratory cost of the tests.

All consultants working on AMU were contacted by email and all junior doctors were informed of the pilot at their induction to AMU and of their role in obtaining verbal consent to test.

Separate discussions were had with all other nursing and clinical aides working on AMU.

Patients were informed of the pilot by posters on the ward and information sheets that were modified to include the routine offer of an HIV test and made available in several different languages,

The admissions proforma was modified to include a routine offer of an HIV test and verbal consent to test for HIV was obtained during admission.

$\mathrm{AP}$ or one of the GU registrars visited the AMU at least once a week during the pilot to remind the consultants and junior staff about the need to offer an HIV test to all patients.

For the 12 months from August 2009-July 2010, we collected data on the number of HIV tests done, the number of positives and the testing rate per consultant team by analysing requests and results from the laboratory. 
From the pilot phase from July 2010 to date we stopped visiting AMU to remind the staff to test and collected the number of tests performed and the positive results as above.

\section{RESULTS}

We looked at three time periods

1. Pre-pilot: September 2008-August 2009, 5484 patients aged 15-59 years were admitted and 205 tests were done-mean of 17 tests per month. $3.7 \%$ of eligible patients tested with four diagnoses made.

2. Pilot: from September 2009-August 2010, 5517 patients aged 15-59 years were admitted and 938 tests were donemean of 78 tests per month. $17.0 \%$ of eligible patients tested with 10 new diagnoses made and one equivocal result. 5/10 had an HIV indicator disease, four were originally from sub Saharan Africa and six had a CD4 count below 350. See table 1 The proportion of patients tested versus those eligible for testing varied between $6 \%$ and $22 \%$ month by month.

The proportion tested by consultant team also exhibited variance between $3 \%$ and $22 \%$

3. Post pilot September 2010-August 2011, 6225 patients aged 15-59 years were admitted and 1399 tests were donemean of 117 tests per month. $22.5 \%$ of eligible patients tested with 15 diagnoses made.

The differences in testing rates between the three time periods were highly statistically significant with $p>0.0001$ between the pre-pilot and the pilot and between the pilot and post pilot time periods ( $\chi^{2}$ using Yates correction).

All patients diagnosed positive were promptly referred as inpatients to the infectious diseases unit for appropriate management.

\section{DISCUSSION}

This pilot study found a higher prevalence of HIV in the population studied (1-3\%) than reported by the HPA in the local population $(0.28 \%)$.

We demonstrated a significant increase in HIV testing rates and subsequent diagnosis but patients may have been offered testing throughout all periods of the study to exclude HIV infection due to the presence of HIV indicator diseases or other reasons. It may have been possible to increase the HIV diagnosis rate by focusing just on indicator diseases but in our pilot 3 out of the 10 diagnosed did not have an indicator condition. Some patients may have known that they were HIV infected but not disclosed this to the admitting doctor, and some patients may have disclosed their status and had this confirmed by testing. The significant increase in testing and positive diagnoses suggest

\begin{tabular}{ll}
$\begin{array}{l}\text { Table } 1 \text { Characteristics of patients diagnosed as HIV positive } \\
(\mathrm{n}=10)\end{array}$ & $\mathbf{n}$ \\
\hline Characteristic & 7 \\
\hline Aged $>45$ years & 7 \\
Male & 3 \\
Female & 3 \\
AIDS defining presentation & 4 \\
Indicator disease at presentation & 4 \\
High prevalence area & 6 \\
CD4 $>200$ & 6 \\
CD4 $>350$ & 9 \\
Survived &
\end{tabular}

that many of the diagnosed patients would have been missed on that admission had their physicians not tested them as part of this pilot.

These patients might well have been diagnosed on a subsequent admission as their disease progressed but later presentation with a lower CD4 count carries a worse prognosis.?

The proportion of patients tested remains low, as many patients who were admitted to the unit having had blood tests done in the Accident and Emergency department and no further bloods were taken before they were discharged.

There also appears to be a large variation of testing between consultants, which suggests the problems lie more with doctors offering tests than patients accepting them. We have provided individual feedback to particular consultants but have no evidence that this changed practice.

The proportion of positive patients who do not come from areas where HIV is endemic (defined for the purposes of this study as being from sub-Saharan Africa) highlights that using country of origin, as additional risk assessment to decide whom not to test is potentially risky.

The ages of patients diagnosed surprised many physicians on AMU, all were over 45 years and 5 were in their 50s. The cohort of patients in the UK is now aging as patients survive longer, and the cut off at aged 59 years for offering testing is probably wrong.

The numbers of patients admitted to AMU over 60 is much greater than those under 60 and the local prevalence of HIV in this population in unknown; however, we have now change the policy on AMU to test up to aged 79 years.

There are currently no data on cost effectiveness of HIV testing in the UK; however, US data suggest testing at an undiagnosed prevalence of $1 / 1000$ would be cost effective. ${ }^{8}$

(Assuming that two out of three individuals with HIV are diagnosed (as was presumed to be the case in 2007 in the UK) then a diagnosed prevalence of $2 / 1000$ would equate to an undiagnosed prevalence of $1 / 1000$.)

Nice guidance published in 2010 states that HIV testing should be offered whenever anyone presents to care in an area of undiagnosed prevalence $>1 / 1000 .^{9}$

Since the pilot concluded the medical director has sent a letter to all consultants emphasising the success of this pilot scheme and instructing all consultants working on AMU to test everybody admitted for HIV.

The AMU pilot in Leicester has demonstrated that HIV testing is acceptable and deliverable and sustainable in a busy medical admissions unit without significant extra resources.

\section{Handling editor Gary Brook.}

Contributors AP lead the pilot and wrote this manuscript. PM is divisional director for acute medicine and helped lead the HIV testing on the AMU and assisted with drafting this manuscript. PS helped with preparation of the manuscript. GMK helped with lab liason and preparation of the manuscript. HF and FN helped draft the manuscript and encouraged and inspired their colleagues on AMU to participate.

Funding This work was supported by a grant from the Department of Health as one of the eight demonstration projects to pilot the expansion of HIV testing outside traditional settings. The funding was used to support the laboratory costs of the extra HIV tests ordered. We would like to thank Mark Ardron, Graham McKinnon, Andy Folwell, Gillian Wardle, Jane Carver and all the medical and nursing staff working on AMU, with a special thanks to all the clinical aides Lynette Barnes, Jason Brightmore, Tina Moorehaines, Jeanette Rutherford, Samantha Tansey, Elizabeth Thomas and Jason Thompson. The assistance received from them during the pilot is greatly appreciated.

Competing interests AP has accepted sponsorship for BMS and Abbott to attend international conferences in line with ABPI guidance. He chaired the 2008 national HIV testing guidelines writing group. 


\section{Clinical}

Ethics approval This was discussed by the local ethics committee who advised that as we were implementing national guidance as part of patients routine care, and that patients were able to opt out of testing, ethics approval was not required.

Provenance and peer review Not commissioned; externally peer reviewed.

\section{REFERENCES}

1 Donaldson L, CMO \& Christine Beasley, CNO Improving the detection and diagnosis of HIV in non-HIV specialties including primary care. Department of Health, 13 September 2007. https://www.cas.dh.gov.uk/ViewandAcknowledgment/ViewAlert. aspx?Alert|D=100818 (accessed 20 Sep 2012).

2 Anon. The UK's appalling failure to tackle HIV. Lancet 2009:373:1820.

3 Lucas SB, Curtis H, Johnson MA. National review of deaths among HIV-infected adults. Clin Med 2008:8:250-2.
4 Sullivan AK, Curtis H, Sabin CA, et al. Newly diagnosed HIV infections: review in UK and Ireland. Br Med J 2005;330:1301-2.

5 British HIV Association.UK national guidelines for HIV testing. 2008. http://www. bhiva.org/documents/Guidelines/Testing/GlinesHIVTest08.pdf (accessed 20 Sep 2012)

6 HIV prevalence data. Health Protection Agency. http://www.hpa.org.uk/web/ HPAwebFile/HPAweb_C/1228207184991 (accessed 20 Sep 2012).

7 HIV in the United Kingdom: 2010 Report. Health Protection Agency, http://www.hpa. org.uk/web/HPAweb\&HPAwebStandard/HPAweb_C/1287145264558 (accessed 20 Sept 2012).

8 Sanders GD, Bayoumi AM, Sundaram V, et al. Cost-effectiveness of screening for HIV in the era of highly active antiretroviral therapy. New Engl J Med 2005;352:570-85.

9 National Institute for Health and Clinical Excellence. Increasing the uptake of HIV testing among men who have sex with men. http://guidance.nice.org.uk/PH34 (accessed 20 Sep 2012). 